\title{
Hippocampal EEG and Unit Activity Responses to Modulation of Serotonergic Median Raphe Neurons in the Freely Behaving Rat
}

\author{
Douglas A. Nitz and Bruce L. McNaughton \\ Arizona Research Laboratories (ARL) \\ Division of Neural Systems, Memory, and Aging (NSMA) \\ University of Arizona \\ Tucson, Arizona 85724 USA
}

\begin{abstract}
Hippocampal EEG, GABAergic interneurons, and principal cells were recorded simultaneously as rats foraged within one of three environments both before and after modulation of serotonergic inputs to the hippocampus. Median raphe microinjections of the 5-HT1a receptor agonist 8-OH-DPAT were made to produce inhibition of serotonergic neurons in this region. Such microinjections produced behavioral arousal and increases in the amplitude of hippocampal EEG theta. Consistent with the pattern of serotonergic innervation of the hippocampus, the GABAergic interneuron population was affected differentially by the microinjections. Principal cells were generally unaffected by the manipulation and maintained robust spatial firing correlates within the foraging environment. The results provide basic data on the relationship between serotonergic median raphe neurons and hippocampal activity in a behaving animal. The data suggest that behavioral responses to manipulation of the serotonergic system are mediated by brain regions other than the hippocampus or are mediated through changes in the activity of hippocampal interneurons.
\end{abstract}

\section{Introduction}

The anatomy of the serotonergic projection from the median raphe nucleus to the hippocam-

${ }^{1}$ Corresponding author. pus in the rat has been well documented (Moore and Halaris 1975; Azmitia and Segal 1978; Vertes and Martin 1988). Serotonergic fibers densely innervate the dentate gyrus region; a more moderate projection innervates the Ammon's horn regions of the hippocampus (Azmitia and Segal 1978). An interesting aspect of this projection is the nearly exclusive innervation of hippocampal GABAergic inhibitory interneurons as opposed to principal cells (Ammon's horn pyramidal and dentate gyrus granule cells) (Freund et al. 1990). Electrophysiological studies in the in vitro slice and in vivo anesthetized preparations are in general agreement with the serotonergic modulation of hippocampal activity through effects on the interneuron population (Segal 1990; Halasy et al. 1992; Ghadimi et al. 1994; Schmitz et al. 1995). Inhibition and excitation of hippocampal interneurons have been observed in response to local application of 5-hydroxytryptamine-1a (5-HT1a) and 5-HT3 receptor agonists, respectively; however, the predominant effect of serotonin and serotonin-releasing agents appears to be an inhibition of hippocampal interneurons by $\mathrm{K}^{+}$-channel modulation through 5 -HT1a receptors (Segal 1990). The innervation of the hippocampal inhibitory network by serotonergic fibers is specific to particular classes of interneuron subtypes. A large subpopulation of hippocampal interneurons that colocalize GABA and parvalbumin are not innervated by the serotonergic system (Miettinen and Freund 1992). Thus, subtype populations of hippocampal interneurons may be affected differentially by the activity of serotonergic neurons.

The activity of serotonergic neurons of the brainstem raphe system has been implicated in the control of both affective and behavioral (sleep/ wake) states. The most robust changes in serotonergic unit activity occur in conjunction with the

LEARNING \& MEMORY 6:153-167 @ 1999 by Cold Spring Harbor Laboratory Press ISSN1072-0502/99 \$5.00

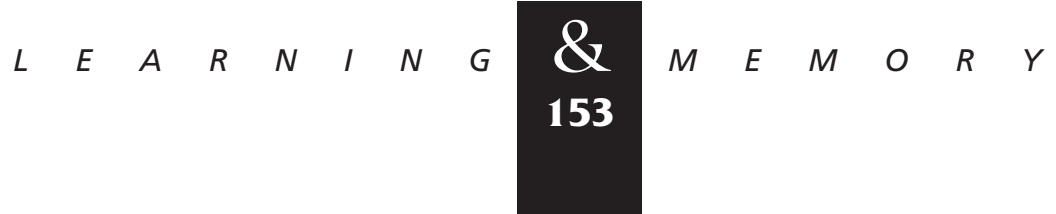


production of sleep/wake states. Discharge of dorsal raphe serotonergic neurons decreases during slow-wave sleep (SWS) relative to waking and is minimal during rapid eye movement (REM) sleep (McGinty and Harper 1976). Accordingly, dorsal raphe microinjection of compounds which inhibit the activity of serotonergic neurons increases REM sleep production (Cespuglio et al. 1979; Portas et al. 1996; Nitz and Siegel 1997). Microinjection of serotonergic agonists within the pons and amygdala either disrupts REM sleep maintenance or disrupts some component of REM sleep (Sanford et al. 1995; Horner et al. 1997).

The utility of drugs acting specifically on the serotonergic system to modulate affective states, such as anxiety and depression, demonstrates the importance of serotonin in modulation of waking processes as well. In this respect, many studies have focused on the relationship between serotonergic neurons of the median raphe nucleus and levels of motor arousal as well as anxiety. Data from both lesion and microinjection experiments suggest that the serotonergic projection from the median raphe to the hippocampus modulates general levels of locomotor activity and anxiety. Median raphe microinjection of the 5-HT1a receptor agonist 8-hydroxy-2-(di-n-propylamino)-tetralin (8OH-DPAT) inhibits discharge of serotonergic neurons and decreases anxiety associated with aversive conditioning and elevated track environments (Hillegaart 1991; File et al. 1996). Increases in locomotor activity associated with the same manipulation have also been observed (Hillegaart 1990). Hippocampal microinjection of 8-OH-DPAT increases anxiety, presumably through action at postsynaptic 5-HT1a receptors (File et al. 1996). Hippocampal lesions and median raphe lesions have each been reported to increase locomotor activity (Jacobs et al. 1975).

Nevertheless, the activity of hippocampal principal cells (Ammon's horn pyramidal and dentate gyrus granule cells) in the freely behaving rat is primarily modulated by the position of the animal within a given environment (O'Keefe and Dostrovsky 1971; McNaughton et al. 1983; Muller et al. 1987). The principal cell population comprises the main output of the hippocampal formation. Thus, behavioral responses to inactivation of serotonergic median raphe neurons remain to be resolved with the activity patterns of hippocampal neurons.

The present study addresses the effects of modulation of the serotonergic system on hippo- campal electroencephalography (EEG), as well as the spatial and temporal activity profiles of hippocampal principal cells and interneurons recorded simultaneously in the behaving animal. Microinjections of the serotonin 5-HT1a autoreceptor agonist 8-OH-DPAT were made in the median raphe nucleus of six rats performing simple foraging tasks in one of three environments. On the basis of the available data, it was predicted that manipulation of the serotonergic system would affect differentially subtypes of GABAergic hippocampal interneurons and that such changes in activity would be associated with increased hippocampal EEG theta amplitude.

\section{Materials and Methods}

\section{SURGICAL PREPARATION}

Surgeries were conducted according to $\mathrm{Na}$ tional Institutes of Health $(\mathrm{NIH})$ guidelines for rodents. Six Fisher-344 rats were anesthetized with sodium pentobarbital and placed in a stereotaxic apparatus. Depth of anesthesia was ensured as necessary with supplemental metafane inhalation. A round, 2-mm-diam. section of skull was removed exposing the neocortex and dura overlying the right hemisphere of the hippocampus. The coordinates of the center of this section were bregma -3.8 and lateral 2.2. After excision of the dura, the outlet of a "hyperdrive" recording apparatus (Gothard et al. 1996) was centered over and lowered to the surface of the exposed neocortex and fixed to the skull with dental cement. Up to five skull screws were inserted across the remaining surface of the skull to aid fixation of the hyperdrive to the skull. Another skull screw was used as ground and was placed over the left frontal cortex.

In each animal, a 30-gauge guide cannula at an anterior/posterior angle of $26^{\circ}$ was targeted for a position $1 \mathrm{~mm}$ above the median raphe nucleus using the following interaural coordinates: anteroposterior +0.8 , lateral 0.0 , dorsoventral +3.0 .

Antibiotic treatment consisted of a presurgical administration of intramuscular bicillin $(0.2 \mathrm{ml})$ and a 1-week postsurgical administration of ampicillin. Analgesic treatment consisted of postsurgical oral administration of $0.8 \mathrm{ml}$ of Children's Tylenol. In addition, a 10\% solution of children's Tylenol was available ad libitum for a period of 3 days after surgery.

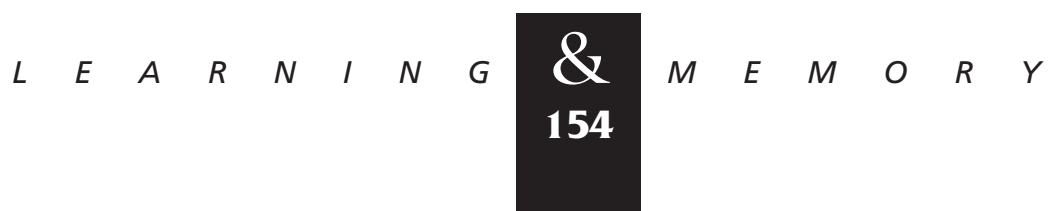




\section{UNIT RECORDINGS}

Fourteen tetrodes (Wilson and McNaughton 1993), each capable of independent movement, were mounted onto a circular array of 14 cannulae (30-gauge guide) housed in a Delrin plastic hyperdrive core. The 14 cannulae were bent slightly to form a circular bundle $1.2 \mathrm{~mm}$ wide at the outlet of the hyperdrive apparatus. Tetrodes were constructed of four $12-\mu \mathrm{m}$ nichrome wires insulated with polyimide. The four wires were twisted together and heat-fused. Tetrode tips were cut flat and gold-plated to produce impedances of 0.3-1.0 $\mathrm{M} \Omega$. Each wire was led to a connector board mounted on the drive that contained an array of pin connections for interface with the recording headstage and cable.

On recording days, a recording headstage and cable were connected to the connector board of the hyperdrive apparatus mounted on the animal. The headstage carried operational amplifiers (unity gain) as well as an array of forward and rear LEDs used for tracking the position of the animal. Signals from the animal were fed, through the headstage and recording cable, to multichannel filter/amplifier modules (Neuralynx, Tucson, AZ) and acquired and digitized $(31.25 \mathrm{kHz})$ by seven synchronized 80486 computers. The rat's position and head orientation (in the horizontal plane) were recorded at 20 frames/sec using the headstage LEDs in conjunction with an SA-2 Dragon tracker (Dragon tracker, Boulder, CO).

Before collection of data, the dorsoventral positions of the individual tetrodes were adjusted to obtain recordings from interneurons throughout subregions of the CA1 and dentate gyrus (DG) areas as well as principal cells in CA1 stratum pyramidale and stratum granulosum of the DG. Activity on each tetrode was referenced to a tetrode placed within the deep neocortex or corpus collosum. Interneurons and principal cells were differentiated by comparison of spike width, average firing rate in quiet waking $(<2 \mathrm{~Hz}$ for principal cells, $>5 \mathrm{~Hz}$ for interneurons), and the presence or absence of complex spike discharge (Fox and Ranck 1973).

Subregions of the CA1 and DG regions were identified by previously described EEG correlates (Bragin et al. 1995; Ylinen et al. 1995), proximity to principal cell layers, and histological analysis of recording electrode tracks. Stratum oriens interneurons were characterized by positively deflecting EEG sharp-wave/ripple events recorded from the same tetrode during sleep. No principal cells were recorded from such tetrode placements, although close proximity to the CA1 pyramidal cell layer was established when subsequent ventral movement of the tetrode encountered principal cells within $400 \mu \mathrm{m}$ of the original tetrode placement. Recordings of interneurons from stratum pyramidale were characterized by concomitant recording of complex-spike pyramidal cells and the presence of flat ripples during sleep. Recordings from stratum radiatum were characterized by negatively deflecting sharp-wave/ripple events and the absence of principal cells. Ventral displacement of the tetrode past the stratum-radiatum produced increases in the amplitude of EEG theta activity. Consistent with movement of the tetrode into the DG stratum moleculare region, further displacement of tetrodes produced large increases in EEG gamma activity before encountering another region (the DG granule cell layer) where multiple principal cells were again encountered.

In the present study, no differences between the responses of CA1 pyramidal and DG granule cells could be discerned by statistical comparison. Similarly, no obvious correlation between the response of interneurons and their identified subregion locale in the hippocampus was observed. As such, pyramidal cells and granule cells, as well as interneurons, were grouped for analysis of drug response properties.

\section{BEHAVIORAL PARADIGMS}

AND MICROINJECTION PROCEDURE

Animals were trained to forage for food reward in one of three environments. Animals were motivated to forage by restricting their food intake such that their weight was maintained at $80-90 \%$ of their free-feeding weight established before training. The three environments included a square chamber $\left(62 \mathrm{~cm}^{2}\right)$ in which animals foraged for randomly scattered chocolate sprinkles; a track 7.5 $\mathrm{cm}$ in width in the shape of an isosceles triangle (75 $\mathrm{cm}$ per side), on which the animal ran in one direction for food reward delivered at the midpoint of each arm; a 93- $\times 43-\mathrm{cm}$ rectangular track, 7.5 $\mathrm{cm}$ in width, on which the animal ran in one direction for food reward delivered at two consecutive corners. Each of the environments was open to several proximal and distal room cues. Each wall of the chamber (62 cm in height) was adorned with large, flat geometric shapes of various size and color.

$$
\begin{array}{lllllllllllllll}
\boldsymbol{Q} & E & A & R & N & I & N & G & M & E & M & O & R & Y \\
155 & & & & & & & & &
\end{array}
$$


Unit activity was first recorded during a 10- to 20-min period in the towel-lined nest area. Subsequently, the animal was displaced to one of the three foraging environments for a 7- to 12-min control recording period. Animals were then returned to the nest area for microinjection. Microinjections $(0.2 \mu \mathrm{l})$ of either saline or $400 \mathrm{ng}$ of $8-\mathrm{OH}-\mathrm{DPAT}$ in saline were made over a 1 -min period. This dose was chosen to match approximately doses used in previous studies from three separate laboratories where robust effects on behavioral variables or EEG theta amplitude had been observed [Hillegaart 1990 ( 1 and 5 g); File et al. 1995 (200 ng); Vertes et al. $1995(0.5,0.75$, and $3.0 \mathrm{~g})$. Preliminary data from three of the animals indicate that the approximate duration of the drug's effects was $40 \mathrm{~min}$, in close agreement with previous reports (Vertes et al. 1995; see Table 3, below). One minute after completion of the microinjection, the injection cannula was removed. The animal was then displaced to the same environment for a second (postmicroinjection) period of foraging.

Typically, at least 3 days separated microinjection experiments on a given animal. On each of these days, the animal spent at least $10 \mathrm{~min}$ foraging in one of the environments such that all experiments were carried out within environments with which the animal was familiar. In a few cases, saline and 8-OH-DPAT microinjections were made on consecutive days to permit analysis of the effects of both saline and 8-OH-DPAT microinjections on activity of the same neurons.

\section{Results}

Successful targeting of microinjection sites to within $1 \mathrm{~mm}$ of the median raphe nucleus was obtained in six animals. Figure 1 depicts a schematic representation of the microinjection sites for each of the animals. On the basis of previous estimates of functional drug diffusion (Martin 1991), microinjections at all but the most dorsal microinjection site would be expected to affect most, if not all, cells carrying 5-HT1a receptors within the median raphe nucleus. Diffusion from the most dorsal microinjection site in Figure 1 would still encompass the majority of the median raphe nucleus. A total of 27 microinjection studies were performed on the six animals. Of these, 19 involved microinjections of the 5-HT1a agonist 8-OHDPAT; eight control vehicle microinjections were made for comparison. On average, the response of hippocampal interneurons to 8-OH-DPAT microin- jections was weakest for the most dorsal microinjection site. This might possibly be explained by a lower effective dose of 8-OH-DPAT reaching the median raphe nucleus or by opposing effects of inactivation of serotonergic cells of the raphe centralis. Nevertheless, hippocampal EEG theta amplitude and some hippocampal interneuron activity rates were clearly affected by microinjections at this site. Therefore, data from this animal were included for statistical analyses.

\section{BEHAVIORAL EFFECTS}

The behavioral effects of the microinjections were in general agreement with those of previous studies (Hillegaart 1990; Vertes et al. 1994). Typically, the first response was for the animal to rise to a standing position and exhibit a general increase in activity characterized by sniffing and scanning in and around the recording bowl. The foraging task was performed robustly by animals after injection. The animal's speed of movement was calculated as the amount of movement (in all directions) per unit time (100 msec) as assessed from the video tracking device. After 8-OH-DPAT microinjection, the average speed with which the animal traversed the environment was increased $12.4 \%$ as compared to the control foraging period of the same day ( $n=19, P<0.01$, paired $t$ test). A decrease of $3.0 \%$ was found after saline microinjections ( $n=8$, $P=0.65$, paired $t$ test) (Fig. 2A).

HIPPOCAMPAL EEG AND THE EFFECTS OF 8-OH-DPAT MICROINJECTIONS

Hippocampal EEG records recorded in the vicinity of the hippocampal fissure were filtered between 6 and $10 \mathrm{~Hz}$. The amplitude of theta activity in the hippocampal EEG was then measured as the deviation (in volts) of identified theta wave peaks from zero. 8-OH-DPAT microinjections increased theta amplitude an average of $21.3 \%$ over control values. Saline microinjections were essentially without effect, resulting on average in $<1 \%$ change in amplitude (Fig. 2B). Paired $t$-tests comparing average theta amplitude in control versus postmicroinjection periods indicated a significant result for 8-OH-DPAT microinjections $(P<0.0001$, $n=19)$ and no effect of saline microinjections $(P=0.75, n=8)$. Theta frequency was essentially unchanged. Less than $4 \%$ change in average theta frequency was observed after both saline and 8-OH-DPAT microinjections.

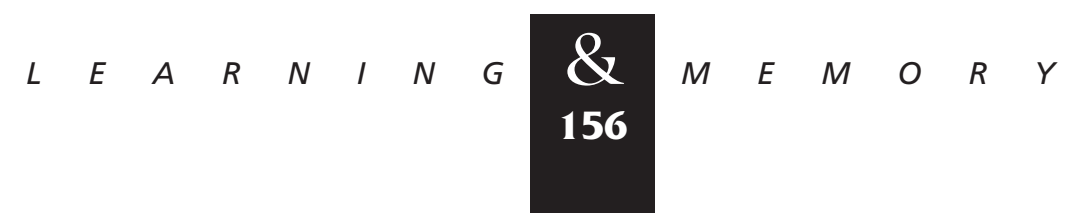



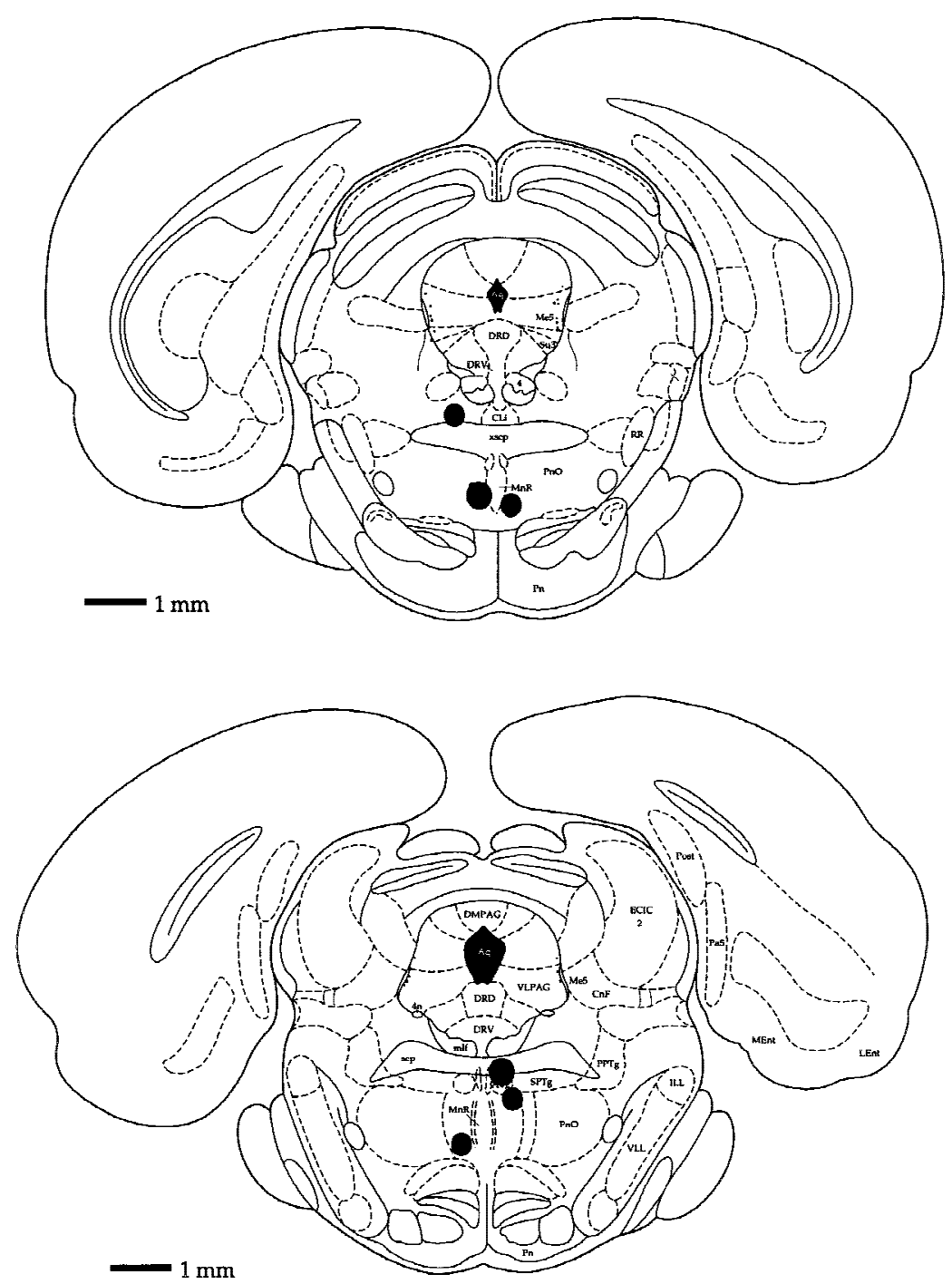

Figure 1: Histological localization of microinjection sites. Solid circles depict histological localization of the microinjection site for each of the six animals. (Top) Bregma, -7.30 mm; interaural, $1.70 \mathrm{~mm}$; (bottom) bregma, $-8 \mathrm{~mm}$; interaural, $1 \mathrm{~mm}$.

Because theta amplitude is correlated positively with the speed with which the animal traverses the environment (Vanderwolf 1969), the effects of changes in movement velocity were controlled for using a velocity-binning process in which the amplitude of each theta wave was binned according to the corresponding speed of movement of the animal (see Fig. 2C). The position tracking record was used to determine the animal's speed of movement within 100-msec periods surrounding each theta peak. For each bin of movement speed, a ratio of average theta amplitude before and after microinjection was calculated. These ratios were then averaged to arrive at an estimate of overall change in theta amplitude controlled for movement speed. When the ratio of the speed $\times$ theta amplitude curves for control versus postmicroinjection sessions was determined, theta amplitude was still found to increase after 8-OH-DPAT (mean ratio of 1.19, 8-OH-DPAT/control) but not saline (mean ratio of 1.022 , saline/ control) microinjections. Comparison of the ratio distributions for the saline and 8-OH-DPAT experiments by analysis of variance (ANOVA) was statistically significant $(P<0.001$, df 1,25$)$. Thus, EEG theta amplitude was increased well above what could be accounted for by changes in movement speed.

EFFECTS OF MICROINJECTIONS ON INTERNEURON ACTIVITIES

As expected, on the basis of differential innervation of the hippocampal inhibitory interneuron network by the serotonergic system, firing rate

$$
\begin{array}{lllllllllllllll}
\hline & E & A & R & N & I & N & G & \begin{array}{c}
\boldsymbol{Q} \\
\mathbf{1 5 7}
\end{array} & M & E & M & O & R & Y
\end{array}
$$


Figure 2: (A) Percentage change in average speed of movement in postmicroinjection foraging periods vs. control foraging periods. Saline, $n=8 ; 8-\mathrm{OH}$ DPAT, $n=19$; (**) $P<0.01$. (B) Percentage change in EEG theta amplitude in postmicroinjection foraging periods versus control foraging periods. Saline, $n=8 ; \quad 8-O H$-DPAT, $\quad n=19 ; \quad(* *)$ $P<0.01$. (C) EEG theta amplitude as a function of movement speed. (๑) Values from control foraging periods; $(\bigcirc)$ values from postmicroinjection foraging periods. 8-OH-DPAT microinjection increased theta amplitude at each movement speed (left); saline microinjection in the same animal (right) was without effect.
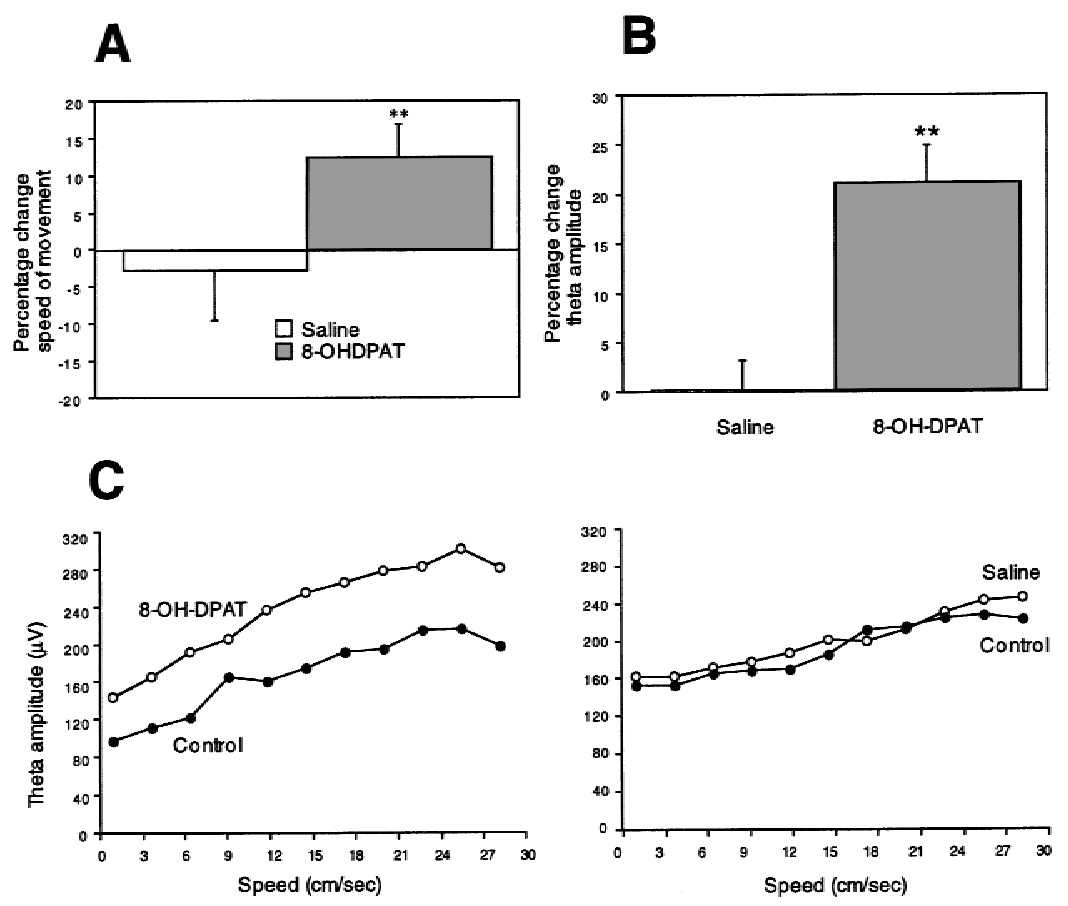

changes were varied across the interneuron population (Fig. 3). The responsivity (or lack of responsivity) of interneurons to 8-OH-DPAT microinjections was not found to depend on their anatomical location within the hippocampus. As such, data obtained from interneurons within stratum oriens ( 7 cells), stratum pyramidale ( 23 cells), stratum radiatum ( 9 cells), stratum moleculare ( 21 cells), and stratum granulosum (8 cells) were pooled for comparison of control versus postmicroinjection activity profiles. Despite the presence of both firing rate reductions and increases (often within the same microinjection experiment), the mean firing rate of the hippocampal interneuron population as a whole increased $20.7 \%$ after microinjection of 8-OH-DPAT as compared to the control foraging session $(24.82 \pm 2.160 \mathrm{~Hz}$ vs. $29.06 \pm 2.360 \mathrm{~Hz}$, $n=48, P<0.001$, paired $t$-test). A mild increase in mean firing rate after saline microinjection was not statistically significant $(24.22 \pm 2.388$ vs. $24.30 \pm 2.383, n=20, P=0.154$, paired $t$-test).

Because the activity of interneurons, like hippocampal EEG theta amplitude, typically shows a strong positive relationship to the velocity with which an animal moves (McNaughton et al. 1983), assessment of the effects of 8-OH-DPAT microinjection on interneuron activity necessitated control for the potentially confounding effects of changes in movement speed. To this end, firing rate in 100msec epochs was binned with respect to the speed of the animal during the same time period as determined by analysis of position information from the tracking apparatus. Plots of firing rate as a function of movement speed were made for each cell and for each experimental session (control vs. saline vs. 8-OH-DPAT) (Fig. 3). For each movement speed bin, a ratio of postmicroinjection versus control firing rate was calculated. The average of these ratios for each cell was taken as the measure of firing rate change in response to the microinjection. Comparison of such speed $\times$ firing rate curves for control sessions versus sessions preceded by microinjection was used to assess the effects of microinjections independent of the effect of microinjections on the speed with which the animal traversed the environment. Note that the shape of the speed $\times$ firing rate curves did not differ between sessions and the lack of overlap for standard error bars of control and 8-OH-DPAT sessions (Fig. 3).

Figure 4 depicts the frequency distribution of percentage change in firing rate (controlled for velocity) for hippocampal interneurons after saline (top graph) and 8-OH-DPAT (bottom graph) microinjections. In comparison with saline microinjections, 8-OH-DPAT microinjections produced a higher percentage of interneurons exhibiting increases or decreases in activity relative to the control foraging session. For 8-OH-DPAT microinjections, the predominant response was an increase in activity. In fact, comparison of the distributions

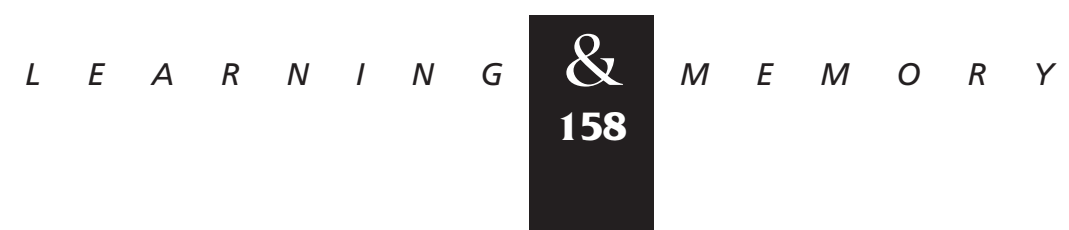


A

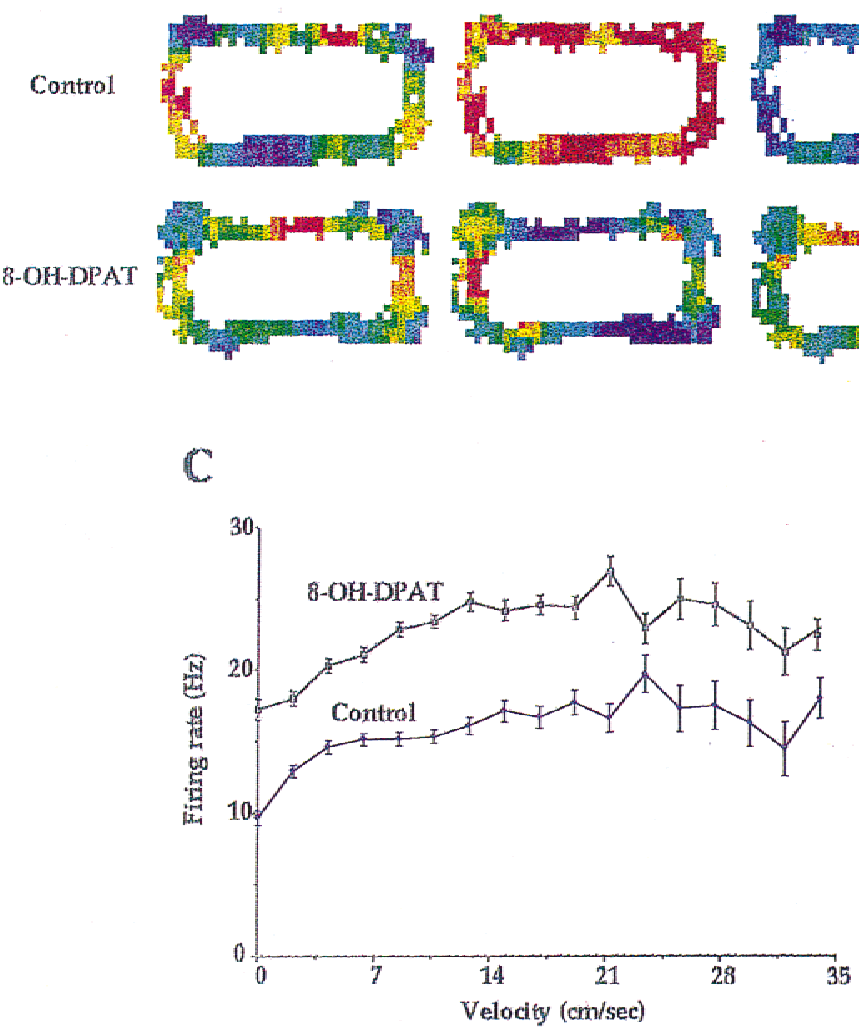

$\mathbb{B}$

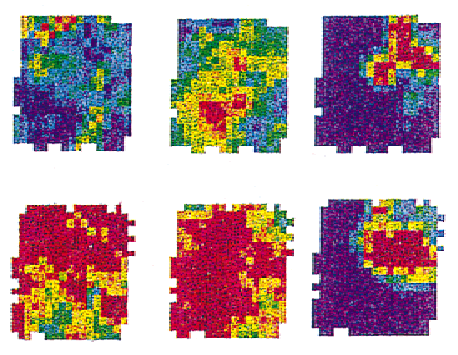

Figure 3: (A) Firing rate for three interneurons recorded simultaneously is depicted as a function of the animal's position with the environment. Dark blue represents low firing rates; dark red represents high firing rates. For cells 1, 2, and 3, dark blue corresponds to firing rates of 11,3, and $22 \mathrm{~Hz}$, respectively. Dark red corresponds to firing rates of 33, 21, and 66 $\mathrm{Hz}$, respectively. For each cell, firing rate maps were generated for control (top plots) and postmicroinjection (bottom plots) foraging periods. Each of these cells exhibited the typical positive relationship between firing rate and speed of movement. Nevertheless, the firing rate of cell 1 is unchanged by the microinjection; that of cells 2 and 3 is decreased and increased, respectively, for the same time period. $(B)$ Firing rate maps for two interneurons and one pyramidal cell (right-most plots) as the animal foraged within a square chamber before (top plots) and after (bottom plots) microinjection of 8-OH-DPAT. Large increases in firing rate were observed for each interneuron. Dark blue corresponds to firing rates of $0 \mathrm{and} 8 \mathrm{~Hz}$, respectively; dark red corresponds to 10 and $24 \mathrm{~Hz}$, respectively, for the two interneurons. A high correlation $(r=0.75)$ was found for the spatial firing patterns of the pyramidal cell before and after drug administration. For this cell, dark blue corresponds to a firing rate of $0 \mathrm{~Hz}$; red corresponds to $4 \mathrm{~Hz}$. (C) Mean firing rate ( \pm S.E.M.) as a function of movement speed is depicted for each of the interneurons of $B$. (Left) Interneuron 1 exhibits a positive relationship between velocity and firing rate under both control and postmicroinjection conditions. Firing rate is increased by 8-OH-DPAT administration at each level of movement speed. (Right) Interneuron 2, a "theta-off" cell, which showed highest firing rates when hippocampal theta was minimal, also exhibits increased discharge after drug administration that is independent of velocity. Nevertheless, this cell maintained an atypical negative relationship between velocity and firing rate.

depicted in Figure 4 by ANOVA approaches statistical significance $(P=0.059$, df 1,66$)$ with percentage change in firing rate, on average, $19.9 \%$ more after 8-OH-DPAT microinjections than after saline microinjections. Thus, the activity of the hippocampal interneuron population as a whole tends to increase after 8-OH-DPAT microinjection even with control for the effects of changes in the animal's speed of movement. In fact, 14 cells exhib- ited rate increases of $>50 \%$. Nevertheless, the effect of the microinjections on interneuron activity is differential, consistent with the wide range of interneuron subtypes found in the hippocampus and the differential innervation of hippocampal interneuron subtypes by the serotonergic system. The standard deviation for the distribution of percentage change after saline microinjections was 0.152 . This value estimates the variability in inter-

$$
\begin{array}{lllllllllllllll}
\hline & E & A & R & N & I & N & G & \begin{array}{c}
\boldsymbol{Q} \\
159
\end{array} & M & E & M & O & R & Y
\end{array}
$$




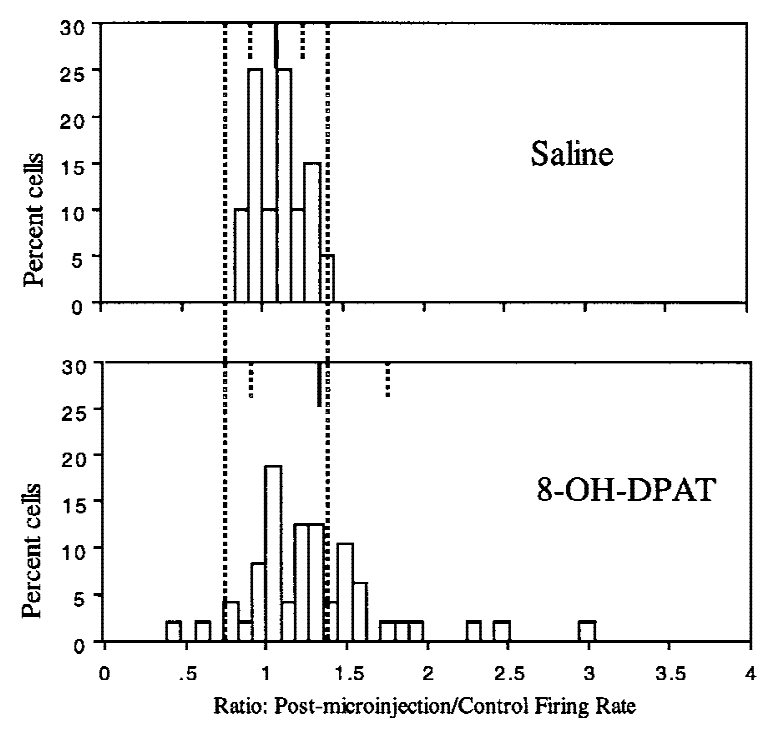

Figure 4: Summary of interneuron responses to $8-\mathrm{OH}$ DPAT and saline microinjections. Distribution of percentage changes in firing rate (controlled for speed of movement) after saline (top) or 8-OH-DPAT (bottom) microinjection. Thin, solid bars at the top of each graph indicate the mean of the distribution. Broken bars indicate \pm 1s.D.. Broken bars crossing both graphs depict \pm 2 S.D. of the postsaline distribution. After 8-OH-DPAT microinjections, the distribution of interneuron responses is flattened relative to the saline distribution indicating the diversity of interneuron responses to the drug treatment. The variance of the two distributions was found to be significantly different using Fisher's test for homogeneity of variance ( $F$ ratio $7.36, P<0.05$, df 47,19$)$.

neuron firing rates as a function of time (one foraging session versus previous or subsequent sessions) and experimental manipulation (vehicle injection effects). After 8-OH-DPAT microinjection, a total of 16 (33.3\%) interneurons exhibited increases in activity greater than twice the value of the standard deviation for percentage change of all cells after saline injection. Using the same criterion, two cells (4.2\%) exhibited decreases in activity.

\section{SPATIAL AND TEMPORAL PATTERNS OF INTERNEURON ACTIVITY AFTER 8-OH-DPAT MICROINJECTIONS}

The spatial firing patterns of hippocampal interneurons before and after microinjection were assessed by comparing correlations between firing rate on a pixel-by-pixel basis for each pair of control and postmicroinjection spatial firing rate maps. As compared with previous studies (McNaughton et al. 1983; Kubie et al. 1987), the spatial firing correlations of interneurons after both saline and 8-OH-DPAT microinjections were fairly high. No difference in control versus postmicroinjection rate map correlations was observed between the saline $(r=0.579 \pm 0.042)$ and 8-OH-DPAT $(r=0.482 \pm 0.033)$ conditions (ANOVA, df 1,66, $P=0.15)$. Despite large changes in overall rate for many interneurons, the spatial pattern of firing was maintained.

The temporal pattern of firing for each cell before and after microinjection was assessed by determining the degree to which the cell tended to fire in a theta rhythm as well as by analysis of the predominant phase of hippocampal EEG theta during which it discharged. Theta modulation was calculated by determining the proportion of firing occurring in each of 10 bins created by parsing the time course of each theta cycle. The proportion of firing in the bin with the highest value was taken as the measure of theta modulation. Across all cells, theta modulation averaged $0.15 \pm 0.005$ (i.e., firing rate was $50 \%$ greater during the phase of peak discharge as compared to overall mean rate). Less than 3\% change in this measure was observed after saline or 8-OH-DPAT microinjections. Each of the 10 theta bins also represents a particular phase of the theta cycle. The theta phase of the bin with the greatest proportion of firing was used to detect potential changes in the phase firing profile of each cell. The average change $\left(34.74 \pm 7.14^{\circ}\right)$ in predominant theta phase discharge between control and 8-OH-DPAT recording periods was not found to be statistically significant.

\section{CORRELATION OF FIRING RATE CHANGES WITH CONTROL SPATIAL AND TEMPORAL FIRING PROFILES}

To some extent, the degree to which a cell exhibited a firing rate response to 8-OH-DPAT microinjection could be determined by spatial and temporal firing characteristics of the cell's behavior during the control foraging session. For this analysis, two additional measures of spatially tuned discharge, spatial information per spike and selectivity (Skaggs et al. 1996), were implemented. Information per spike (Skaggs et al. 1996) reflects the number of bits of information concerning the animal's position in the environment contained in each spike discharge. Selectivity (Skaggs et al. 1996) is the ratio of the highest and lowest firing

$$
\begin{array}{lllllllllllllll}
L & E & A & R & N & I & N & G & \underset{160}{\boldsymbol{Z}} & M & E & M & O & R & Y
\end{array}
$$


rates in the spatial firing rate map. Table 1 lists correlations between spatial/temporal discharge parameters measured during the control foraging session and subsequent drug response (expressed as percentage change in velocity-controlled firing rate in drug vs. control sessions). Also included in Table 1 are correlations of the same parameters with firing rate during REM sleep; these correlations are based on a subset (32) of the 48 cells for which a drug response was characterized (REM sleep data were not available for all recording sessions). Many of the variables considered in Table 1 are highly correlated. In general, cells with high mean firing rates in REM sleep and during foraging sessions exhibit less spatially modulated discharge and less theta modulation. In this data set, such cells were unlikely to exhibit either an increase or decrease in firing rate in response to 8-OH-DPAT microinjection (Table 1B).

\section{CORRELATION OF FIRING RATE CHANGES} WITH SPATIAL/TEMPORAL PROFILE CHANGES

Percentage changes in firing rate after microinjections of 8-OH-DPAT were correlated with changes in spatial (information/spike and selectivity) and temporal (theta modulation, theta phase preference) firing parameters. On the basis of the correlations between mean rate and spatial parameters in Table 1A, changes in spatial firing characteristics might be expected to follow changes in mean rate. This was indeed the case; percentage change in firing rate was significantly correlated with percentage change in information/spike $(r=-0.418, P<0.005)$ and selectivity $(r=-0.486$, $P<0.001)$.
In the control session, theta modulation of interneuron discharge had a negative relationship to mean firing rate (Table 1 ). Nevertheless, a correlation between changes in theta modulation and firing rate in response to 8-OH-DPAT microinjection was not observed ( $r=0.083, P>0.4)$. Unlike spatial firing parameters, the rhythmicity with which an interneuron discharges did not undergo change correlated with firing rate changes as expected from the significant correlation between these variables in the control session.

\section{PRINCIPAL CELLS}

Overall, little change was observed in the discharge properties of hippocampal pyramidal and granule cells during foraging sessions after saline or 8-OH-DPAT microinjections as compared to control foraging sessions. Table 2 lists mean values for spatial discharge parameters, mean firing rate, and degree of theta modulation for all principal cells during control and postmicroinjection periods. Paired $t$ tests for both the saline and 8-OH-DPAT microinjection conditions did not reach statistical significance for any of the variables.

Table 2 also lists mean spatial firing rate map correlations for all principal cells. The spatially modulated discharge of principal (place) cells was essentially unchanged. Mean spatial firing rate map correlations of postmicroinjection and control foraging sessions did not differ between the saline and 8-OH-DPAT microinjection conditions (ANOVA, df $1,86, P=0.12$ ). Thus, stable place-specific firing was observed under conditions when firing rates of many inhibitory interneurons had changed dramatically.

Table 1: Parameter correlations

\begin{tabular}{|c|c|c|c|c|c|}
\hline & Info./spike & Selectivity & Mean rate & REM rate & Theta Modulation \\
\hline \multicolumn{6}{|c|}{ A. Control session spatial and temporal firing descriptors } \\
\hline Info./spike & 1.0 & $0.907^{* * * *}$ & $-0.443^{* * *}$ & $-0.596 * * *$ & 0.275 \\
\hline Selectivity & & 1.0 & $-0.481^{* * *}$ & $-0.536^{* *}$ & 0.252 \\
\hline Mean rate & & & 1.0 & $0.872^{* * * *}$ & $-0.407 * *$ \\
\hline REM rate & & & & 1.0 & $-0.383^{*}$ \\
\hline Theta modulation & & & & & 1.0 \\
\hline \multicolumn{6}{|c|}{ B. Control session spatial/temporal firing descriptors and subsequent drug response } \\
\hline $\begin{array}{l}\text { Drug response } \\
\quad(\% \text { change in firing rate })\end{array}$ & $0.430 * *$ & $0.408^{* *}$ & $-0.399 * *$ & -0.409 & -0.037 \\
\hline
\end{tabular}

(*) $P<0.05 ;(* *) P<0.01 ;(* * *) P<0.001 ;(* * * *) P<0.0001$.

$\begin{array}{lllllllllllllll}L & E & A & R & N & I & N & G & \begin{array}{c}\mathbf{Z} \\ \mathbf{1 6 1}\end{array} & M & E & M & O & R & Y\end{array}$


Table 2: Principal cell discharge characteristics following saline and 8-OH-DPAT microinjections

\begin{tabular}{|c|c|c|c|c|}
\hline & $\begin{array}{l}\text { Control } \\
\text { (saline) }\end{array}$ & $\begin{array}{l}\text { Saline } \\
(n=21)\end{array}$ & $\begin{array}{l}\text { Control } \\
\text { (DPAT) }\end{array}$ & $\begin{array}{c}\text { DPAT } \\
(n=67)\end{array}$ \\
\hline Info/spike (bits/spike) & $1.267(0.178)$ & $1.263(0.120)$ & $1.190(0.138)$ & $1.123(0.136)$ \\
\hline Selectivity & $16.74(3.886)$ & $16.20(3.667)$ & $13.37(2.568)$ & $12.13(1.957)$ \\
\hline In-field firing rate $(\mathrm{Hz})$ & $4.263(1.315)$ & $3.543(0.776)$ & $3.262(0.588)$ & $3.373(0.736)$ \\
\hline Mean firing rate $(\mathrm{Hz})$ & $0.589(0.101)$ & $0.633(0.082)$ & $0.773(0.170)$ & $0.759(0.149)$ \\
\hline Theta modulation & $0.184(0.012)$ & $0.181(0.014)$ & $0.183(0.007)$ & $0.182(0.007)$ \\
\hline $\begin{array}{l}\text { Rate map correlation ( } r \text { ) } \\
\text { (vs. control session) }\end{array}$ & & $0.684(0.045)$ & & $0.583(0.060)$ \\
\hline \multicolumn{2}{|c|}{ Theta phase (Idrug - controll degrees) } & $55.80(11.88)$ & & $59.76(7.20)$ \\
\hline
\end{tabular}

All values expressed as mean (S.E.M.).

Finally, an assessment was made as to the effects of 8-OH-DPAT microinjections on theta phase precession of principal cell discharge. O'Keefe and Recce (1993) and later Skaggs et al. (1996) demonstrated that the discharge of principal cells precesses from predominantly late to early phases of hippocampal EEG theta as the animal passes through the place field of a cell. Although a quantitative analysis of this phenomena was not attempted in the present study, all principal cells that were observed to exhibit phase precession during control foraging sessions were also found to exhibit phase precession after median raphe microinjection of 8-OH-DPAT.

EFFECTS OF 8-OH-DPAT MICROINJECTION

ON PRODUCTION OF SLEEP/WAKE STATES

The effects of 8-OH-DPAT microinjections on the production of specific sleep/wake states were determined for three of the animals. After microinjection of 8-OH-DPAT or saline, animals remained in the towel-lined bowl for a 3 -hr recording period. Consistent with the increased behavioral activation observed within the foraging environment, $8-\mathrm{OH}-$ DPAT microinjections increased time spent awake during the first $40 \mathrm{~min}(n=3, P<0.05$, repeated measures ANOVA) postmicroinjection for each of the three subjects (Table 3 ). Increased wake time was offset by decreases in both SWS and REM sleep time with time spent in SWS significantly decreased over the first $20 \mathrm{~min}$ after 8-OH-DPAT microinjection $(n=3, P<0.05)$.

\section{Discussion}

As observed previously, 8-OH-DPAT microinjections into the median raphe region produced

Table 3: Sleep/wake state production following saline and 8-OH-DPAT microinjections

\begin{tabular}{llcrr}
\hline & Time postinjection & SWS & REM & Wake \\
\hline Saline & hr 1 & $38.6(1.5)$ & $8.1(2.5)$ & $13.3(3.2)$ \\
& $(0-20 \mathrm{~min})$ & $13.8(0.8)$ & $1.3(0.7)$ & $4.8(0.9)$ \\
& $(21-40 \mathrm{~min})$ & $13.3(1.4)$ & $3.3(0.6)$ & $3.4(2.0)$ \\
& $(41-60 \mathrm{~min})$ & $11.3(1.2)$ & $3.4(1.3)$ & $5.3(1.2)$ \\
& hr 2 & $36.3(6.0)$ & $7.6(0.5)$ & $16.7(5.6)$ \\
hr 3 & $29.3(8.2)$ & $3.3(1.8)$ & $27.3(7.9)$ \\
8-OH-DPAT & & & \\
& hr 1 & $34.1(4.1)$ & $3.9(1.4)$ & $21.9(2.9)$ \\
& $(0-20 \mathrm{~min})$ & $9.3(2.0)^{*}$ & $1.3(0.7)$ & $9.4(1.4)^{*}$ \\
& $(21-40 \mathrm{~min})$ & $10.7(2.0)$ & $0.3(0.3)$ & $9.0(2.1)^{*}$ \\
& $(41-60 \mathrm{~min})$ & $14.2(0.4)$ & $7.8(5.3)$ & $3.5(0.6)$ \\
& hr 2 & $34.7(3.3)$ & $6.1(3.0)$ & $19.2(5.5)$ \\
& hr 3 & $37.9(3.0)$ & $4.8(2.4)$ & $17.3(5.0)$ \\
\hline
\end{tabular}

$n=3 ;\left(^{*}\right) P<0.05$. All values as mean minutes (S.E.M.).

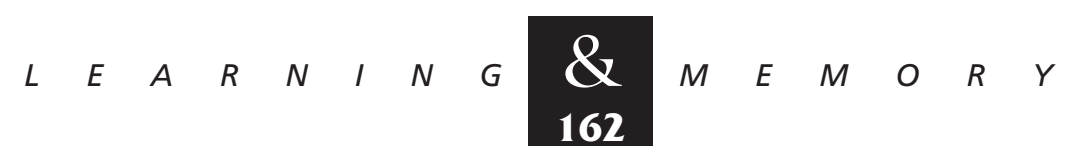


increases in overall speed of movement (Hillegaart 1990) through the environment as well as pronounced changes in EEG theta amplitude (Vertes et al. 1994). Slightly smaller median raphe microinjections of the same compound have been reported previously to produce anxiolytic responses (File et al. 1995). In this study, 8-OH-DPAT microinjections were also found to increase significantly the amount of time spent awake over a 40-min period, providing some indication as to the duration of the drug's action. The dissipation of the drug's effect after $\sim 40 \mathrm{~min}$ is in reasonably close agreement with previous studies (Vertes et al. 1994).

In vitro studies indicate that this compound produces direct inhibitory effects on median and dorsal raphe serotonergic neurons (Sprouse and Aghajanian 1987, 1988; Sinton and Fallon 1988). Serotonergic cells in more caudal regions of the raphe system have been characterized repeatedly by slow discharge rate, long duration action potentials, and near cessation of firing during REM sleep (for review, see Jacobs and Azmitia 1992). Such cells are inhibited by systemic administration of 5-HT1a receptor agonists, which presumably produce inhibition through agonist action at 5-HT1a autoreceptors. In freely behaving cats, systemic administration of nonselective 5-HT receptor agonists was found to inhibit median raphe cells characterized by the aforementioned criteria (Rasmussen et al. 1984). Other cell types within the median raphe were generally unresponsive. Of course, direct effects of this compound on nonserotonergic neurons of the median raphe region cannot be ruled out completely. Nevertheless, it is reasonable to assume that serotonergic median raphe neurons were inhibited after 8-OH-DPAT microinjections and that this inhibition was relatively selective. The interpretation of the results is offered with this in mind.

Within this context, it should be noted that the present experimental paradigm was chosen for its potential to clarify the global effect of median raphe serotonergic unit discharge modulation on hippocampal activity, as opposed to the direct hippocampal delivery of compounds that affect only a single type of 5-HT receptor or are restricted to action within a particular subregion of the hippocampal complex. Manipulations at the level of the cells of origin should result in a modulation of serotonergic activity, while preserving the balance of 5-HT receptor subtype stimulation within the hippocampus.

\author{
SEROTONERGIC MODULATION \\ OF SLEEP/WAKE STATE
}

Although the data concerning the awakening effects of median raphe microinjections of 8-OHDPAT are based on only a few animals, they do suggest a role for the median raphe in the regulation of sleep and wake state production. Surprisingly, 8-OH-DPAT microinjection within the median raphe produced increases in wake time. Previous studies have demonstrated that acute inactivation of the dorsal raphe serotonergic neurons by cooling or by microinjection of 8-OH-DPAT or the GABA-A receptor agonist muscimol increases time spent in REM sleep and decreases locomotor activity (Cespuglio et al. 1979; Hillegaart 1990; Portas et al. 1996; Nitz and Siegel 1997). At least with regard to regulation of behavioral arousal, the present data suggest potentially opposite roles for median and dorsal serotonergic raphe neurons. The present data are consistent with the finding that median, but not dorsal, raphe lesions produced increases in locomotion (Jacobs et al. 1974). Such differences in function are most likely rooted in the divergence of median versus dorsal raphe projection patterns. In particular, neurons of the dorsal raphe nucleus project more heavily to regions of the dorsolateral pons involved in the production of REM sleep. Confirmation of this finding would signal the need to consider separately the dorsal and median raphe serotonergic neurons when evaluating behavioral or neurophysiological responses to systemically administered compounds with action involving the serotonergic system.

\section{SEROTONERGIC MODULATION OF HIPPOCAMPAL EEG}

Consistent with data obtained in anesthetized preparations, 8-OH-DPAT microinjections into the median raphe produced large increases in the amplitude of EEG theta activity as the animal traversed the environment. These increases were independent of changes in the animal's speed of movement.

The most likely explanation for the increase in EEG theta amplitude after 8-OH-DPAT microinjections is the associated increase in interneuron discharge rates. Production of theta rhythmicity in the hippocampal EEG is, in part, a function of rhythmic GABAergic inhibitory postsynaptic potentials impinging on principal cells of the Ammon's horn

$$
\begin{array}{lllllllllllllll} 
& E & A & R & N & I & N & G & \begin{array}{r}
\boldsymbol{Q} \\
163
\end{array} & M & E & M & O & R & Y
\end{array}
$$


and dentate gyrus regions (Buzsaki et al. 1983). In the present study, interneurons exhibiting increases in discharge rates in response to $8-\mathrm{OH}-$ DPAT microinjections maintained proportional unit discharge rates across the EEG theta wave cycle similar to those observed during control sessions. What small changes in theta modulation of unit discharge did occur after 8-OH-DPAT microinjection were not correlated with overall changes in firing rate. Nevertheless, the absolute difference in highest versus lowest discharge rate across the EEG theta wave cycle was increased or decreased in proportion to the increase or decrease in overall mean firing rate. Thus, an increase in the net difference in GABAergic interneuron discharge between positive and negative phases of the EEG theta rhythm is produced by median raphe microinjection of 8-OH-DPAT. This difference most likely accounts for the observed increases in EEG theta amplitude. In this respect, it is notable that average increases in interneuron discharge rate and EEG theta amplitude were each $\sim 20 \%$.

Alterations in the activity of septal cholinergic or GABAergic afferents to the hippocampus most likely contributed to the 8-OH-DPAT-induced increase in EEG theta amplitude. The septal nucleus is an integral component for EEG theta production in the hippocampus, and median raphe serotonergic neurons densely innervate this nucleus (Conrad et al. 1974; Azmitia and Segal 1978; for review, see Bland 1986; Vertes and Kocsis 1997). Median raphe stimulation disrupts rhythmic firing of septal neurons (Assaf and Miller 1978). Inhibition of serotonergic inputs to the septum by median raphe 8-OH-DPAT microinjection produces rhythmic theta frequency discharge of septal neurons (Kinney et al. 1996). Further studies aimed at determining the response, in behaving animals, of septal cholinergic and GABAergic neurons to inactivation of median raphe serotonergic neurons will be necessary to parcel out the potentially complex modulation of hippocampal activity patterns by the raphe system.

\section{SEROTONERGIC MODULATION OF HIPPOCAMPAL INTERNEURON ACTIVITIES}

After median raphe microinjection of $8-\mathrm{OH}-$ DPAT, hippocampal interneurons typically exhibited increases in discharge rate, although a few cells exhibited large reductions in activity. Consistent with the presence of interneurons innervated by serotonergic fibers in all subregions of the hip- pocampus, no obvious correlation between interneuron response and anatomical location was found. Modulation of interneuron discharge rate was independent of changes in locomotor behavior. Measures of interneuron spatial and temporal firing patterns were little affected by the microinjections.

Both direct and indirect effects of inactivation of serotonergic afferents to the hippocampus may be responsible for the changes in discharge rate of interneurons. Serotonergic neurons of the median raphe nucleus innervate directly the hippocampal interneuron population. In the in vitro slice preparation, application of serotonin produces inhibition of interneurons (Segal 1990; Schmitz et al. 1995). Thus, the results of the present study are compatible with direct disinhibition of the hippocampal interneuron population after inactivation of hippocampally projecting serotonin neurons of the median raphe nucleus. An indirect action through hippocampally projecting septal neurons may also contribute to the observed alterations of hippocampal interneuron discharge. Hippocampally projecting GABAergic neurons of the septum innervate exclusively the GABAergic interneuron population of the hippocampus (Freund and Antal 1988; Gulyas et al. 1990), and median raphe microinjection of 8-OH-DPAT has been shown to induce rhythmic discharge in septal neurons in the anesthetized rat (Kinney et al. 1996).

The present findings indicate that serotonin affects differentially the discharge of specific hippocampal interneuron subclasses and that these subclasses may share spatial and temporal discharge characteristics in the freely behaving animal. GABAergic cells of the hippocampus, which express parvalbumin, do not receive synaptic innervation by serotonergic afferents (Miettinen and Freund 1992); many parvalbumin-positive interneurons of the hippocampus have been characterized as basket cells (Katsumaru et al. 1988). Although speculative, it is possible that this cell group also exhibits high wake and REM sleep firing rates, relatively less theta modulation, and low spatial selectivity in the intact rat, as such cells were least likely to respond to manipulation of the median raphe serotonergic cells.

The baseline data collected in this study demonstrate that the extent to which an interneuron's discharge is theta modulated is predictive of baseline firing rate; high rates of discharge are significantly correlated with low theta rhythmicity. Nevertheless, the rhythmicity with which hippocam-

$$
\begin{array}{lllllllllllllll} 
& E & A & R & N & I & N & G & \begin{array}{r}
\boldsymbol{Q} \\
164
\end{array} & M & E & M & O & R & Y
\end{array}
$$


pal interneurons discharge was essentially unaffected by median raphe 8-OH-DPAT microinjections, which changed drastically interneuron firing rates. Thus, although firing rate is predictive of rhythmicity, the cellular properties underlying these features are either unrelated or respond differentially to modulation of serotonergic input.

\section{SEROTONERGIC MODULATION OF HIPPOCAMPAL PRINCIPAL CELL ACTIVITIES}

Principal cells of stratum pyramidale and stratum granulosum were generally unresponsive to the microinjections. No changes in firing rate were observed for this cell class. Because this cell type was most often recorded in conjunction with interneurons, the lack of principal cell response was dissociated from interneurons, which did respond as well to changes in EEG theta amplitude and the animal's speed of movement. Place-specific discharge of principal cells was essentially unchanged as assessed by correlation of spatial firing rate maps before and after microinjections as well as two additional measures of place-specific activity. Greater responsivity of hippocampal interneurons than principal cells to activation of hippocampal 5-HT receptors has been observed previously (Segal 1990; Schmitz et al. 1995). Given that a large proportion of the interneuron population responded robustly to median raphe 8 -OH-DPAT microinjection, this lack of principal cell response was surprising. The result indicates that despite high interconnectivity of the interneuron and principal cell populations, the activity patterns of these two main classes of cell type in the hippocampus can be dissociated. A similar dissociation was observed by Mizumori et al. (1989) who observed a decline in CA1 interneuron firing rates and a suppression of EEG theta amplitude after injection of a local anesthetic into the medial septum, with no effect on CA1 place cells.

Principal cells of the hippocampus comprise the main source of efferents from the hippocampus. As such, it is difficult to reconcile the lack of principal cell response to the 8-OH-DPAT microinjections with hypotheses suggesting that the hippocampus mediates behavioral responses to manipulation of serotonergic neurons (Jacobs et al. 1975; File et al. 1996). It is possible that higher doses of 8-OH-DPAT might have produced change in the activity of principal cells or that the anxiolytic effects of median raphe inactivation are mediated by the ventral hippocampus. Higher doses of 8-OH-DPAT were not evaluated in this set of experiments, but the dosage used lies between previously used doses shown to produce anxiolytic effects (Hillegaart 1991; File et al. 1996). Ventral hippocampal recordings were not made, but previous studies have demonstrated an anxiogenic response to dorsal hippocampal microinjection of 8-OH-DPAT (File et al. 1996). Thus, a likely scenario is that behavioral effects of 8-OH-DPAT median raphe microinjections are mediated by other brain structures. Alternatively, it is conceivable that hippocampal interneurons could mediate behavioral responses directly. A subpopulation of hippocampal GABAergic neurons do project to the septum (Alonso and Kohler 1982). Calbindin-positive GABAergic neurons of the hippocampus are heavily innervated by the serotonergic system and comprise the main source of the hippocampal GABAergic projection to the septum (Toth and Freund 1992; Freund and Buzsaki 1996).

The present findings demonstrate that $8-\mathrm{OH}-$ DPAT microinjections into the median raphe nucleus of freely behaving rats increased time spent awake, altered the discharge rate of hippocampal interneurons, increased hippocampal EEG theta amplitude, and increased speed of movement during a simple foraging task. Changes in hippocampal interneuron firing rates were independent of changes in behavioral arousal and could account for increased EEG theta amplitude. Firing rate and spatially tuned activity of hippocampal principal cells were maintained at control levels under the same experimental conditions. Inclusion of recordings from the septal nucleus, under similar conditions, may reveal a role of this structure in production of EEG theta amplitude changes and possibly behavioral effects of manipulations affecting serotonergic median raphe neurons.

\section{Acknowledgments}

This study was supported by U.S. Public Health Service (PHS) grant NS20331 and postdoctoral national Research Service Award (NRSA) NS10046.

The publication costs of this article were defrayed in part by payment of page charges. This article must therefore be hereby marked "advertisement" in accordance with 18 USC section 1734 solely to indicate this fact.

\section{References}

Alonso, A. and C. Kohler. 1982. Evidence for separate projections of hippocampal pyramidal and non-pyramidal neurons to different parts of the septum in the rat brain. Neurosci. Lett. 31: 209-214.

$$
\begin{array}{lllllllllllllll} 
& E & A & R & N & I & N & G & \begin{array}{c}
\boldsymbol{Q} \\
165
\end{array} & M & E & M & O & R & Y
\end{array}
$$




\section{Nitz and McNaughton}

Assaf, S.Y. and J.J. Miller. 1978. The role of a raphe serotonin system in the control of septal unit activity and hippocampal desynchronization. Neuroscience 3: 539-550.

Azmitia, E.C. and M. Segal. 1978. An autoradiographic analysis of the differential ascending projections of the dorsal and median raphe nuclei in the rat. J. Comp. Neurol. 179: $641-668$.

Bland, B.H. 1986. The physiology and pharmacology of hippocampal formation theta rhythms. Prog. Neurobiol. 26: $1-54$.

Bland, B.H., P. Andersen, T. Ganes, and O. Sveen. 1980. Automated analysis of rhythmicity of physiologically identified hippocampal formation neurons. Exper. Brain Res. 38: 205-219.

Bragin, A., G. Jando, Z. Nadasdy, J. Hetke, K. Wise, and G. Buzsaki. 1995. Gamma (40-100 Hz) oscillation in the hippocampus of the behaving rat. J. Neurosci. 15: 47-60.

Buzsaki, G., L. Leung, and C.H. Vanderwolf. 1983. Cellular basis of hippocampal EEG in the behaving rat. Brain Res. Rev. 6: 139-171.

Cespuglio, R., M.E. Gomez, E. Walker, and M. Jouvet. 1979. Effets du refriodissement et de la stimulation des noyaux du systeme du raphe sur les etats du vigilance chez le chat. Electroencephalogr. Clin. Neurophysiol. 47: 289-308.

File, S.E., L.E. Gonzalez, and N. Andrews. 1996. Comparative study of pre- and postsynaptic 5-HT1a receptor modulation of anxiety in two ethological animal tests. J. Neurosci. 16: 4810-4815.

Fox, S.E. and J.B. Ranck Jr. 1973. Localization and anatomical identification of theta and complex-spike cells in dorsal hippocampal formation of rats. Exp. Neurol. 49: 299-313.

Freund, T.F. and M. Antal. 1988. GABA-containing neurons in the septum control inhibitory interneurons in the hippocampus. Nature 336: 170-173.

Freund, T.F. and G. Buzsaki. 1996. Interneurons of the hippocampus. Hippocampus 6: 347-470.

Freund, T.F., A.I. Gulyas, L. Acsady, T. Gorcs, and K. Toth. 1990. Serotonergic control of the hippocampus via local inhibitory interneurons. Proc. Natl. Acad. Sci. 87: 8501-8505.

Ghadimi, B.M., W. Jarolimek, and U. Misgeld. 1994. Effects of serotonin on hilar neurons and granule cell inhibition in the guinea pig hippocampal slice. Brain Res. 633: 27-32.

Gothard, K.M., W.E. Skaggs, and B.L. McNaughton. 1996. Dynamics of mismatch correction in the hippocampal ensemble code for space: Interaction between path integration and environmental cues. J. Neurosci. 16: $8027-8040$.
Gulyas, A.I., T.J. Gorcs, and T.F. Freund. 1990. Innervation of different peptide-containing neurons in the hippocampus by GABAergic septal afferents. Neuroscience 37: 31-41.

Halasy, K., R. Mietingen, E. Szabat, and T. Freund. 1992. GABAergic interneurons are the major postsynaptic targets of median raphe afferents in the rat dentate gyrus. Eur. J. Neurosci. 4: 144-153.

Hillegaart, V. 1990. Effects of local application of 5-HT and 8-OH-DPAT into the dorsal and median raphe nuclei on motor activity in the rat. Phys. Behav. 48: 143-148.

1991. Functional topography of brain serotonergic pathways in the rat. Acta Physiol. Scand. Suppl. 142: 1-54.

Horner, R.L., L.D. Sanford, D. Annis, A.I. Pack, and A.R. Morrison. 1997. Serotonin at the laterodorsal tegmental nucleus suppresses rapid-eye-movement sleep in freely behaving rats. J. Neurosci. 17: 7541-7552.

Jacobs, B.L. and E.C. Azmitia. 1992. Structure and function of the brain serotonin system. Physiol Rev. 72: 165-229.

Jacobs, B.L., W.D. Wise, and K.M. Taylor. 1974. Differential behavioral and neurochemical effects following lesions of the dorsal or median raphe nuclei in rats. Brain Res.

79: $353-361$.

Jacobs, B.L., C. Trimbach, E.E. Eubanks, and M. Trulson. 1975. Hippocampal mediation of raphe lesion and PCPA-induced hyperactivity in the rat. Brain Res. 94: 253-261.

Katsumaru, H., T. Kosaka, C.W. Heizmann, and K. Hama. 1988. Immunocytochemical study of GABAergic neurons containing the calcium-binding protein parvalbumin in the rat hippocampus. Exp. Brain Res. 72: 347-362.

Kinney, G.G., B. Kocsis, and R.P. Vertes. 1995. Injections of muscimol into the median raphe nucleus produce hippocampal theta rhythm in the urethane anesthetized rat. Psychopharmacology 120: 244-248.

1996. Medial septal unit firing characteristics following injections of 8-OH-DPAT into the median raphe nucleus. Brain Res. 708: 116-122.

Kubie, J.L., R.U. Muller, and E. Bostock. 1990. Spatial firing properties of hippocampal theta cells. J. Neurosci. 10: $1110-1123$.

Martin, J.H. 1991. Autoradiographic estimation of the extent of reversible inactivation produced by microinjection of lidocaine and muscimol in the rat. Neurosci. Lett. 127: 160-164.

McNaughton, B.L., C.A. Barnes, and J. O'Keefe. 1983. The contributions of position, direction, and velocity to single unit activity in the hippocampus of freely-moving rats. Exp. Brain Res. 52: 41-49.

Miettinen, R. and T.F. Freund. 1992. Convergence and 
segregation of septal and median raphe inputs onto different subsets of hippocampal inhibitory interneurons. Brain Res. 594: $263-272$.

Mizumori, S.J., C.A. Barnes, and B.L. McNaughton. 1989. Reversible inactivation of the medial septum: Selective effects on the spontaneous activity of different hippocampal cell types. Brain Res. 500: 99-106.

Moore, R.Y. and A.E. Halaris. 1975. Hippocampal innervation by serotonin neurons of the midbrain raphe in the rat. J. Comp. Neurol. 164: 171-184.

Muller, R.U., J.L. Kubie, and J.L. Ranck Jr. 1987. Spatial firing patterns of hippocampal complex-spike cells in a fixed environment. J. Neurosci. 7: 1935-1950.

Nitz, D. and J. Siegel. 1997. GABA release in the dorsal raphe nucleus: Role in the control of REM sleep. Am. J. Physiol. 273: 8451-8455.

O'Keefe, J. and J. Dostrovsky. 1971. The hippocampus as a spatial map. Preliminary evidence from unit activity in the freely moving rat. Brain Res. 34: 171-175.

O'Keefe, J. and M.L. Recce. 1993. Phase relationship between hippocampal place units and the EEG theta rhythm. Hippocampus 3: 317-330.

Portas, C.M., M. Thakkar, D. Rainnie, and R.W. McCarley. 1996. Microdialysis perfusion of

8-hydroxy-2-(di-n-propylamino)-tetralin (8-OH-DPAT) in the dorsal raphe nucleus decreases serotonin release and increases rapid-eye-movement sleep in the freely moving cat. J. Neurosci. 16: 2820-2828.

Rasmussen, K., J. Heym, and B.L. Jacobs. 1984. Activity of serotonin-containing neurons in nucleus centralis superior of freely moving cats. Exp. Neurol. 83: 302-317.

Sanford, L.D., S.M. Tejani-Butt, R.J. Ross, and A.R. Morrison. 1995. Amygdaloid control of alerting and behavioral arousal in rats: Involvement of serotonergic mechanisms. Arch. Ital. Biol. 134: 81-99.

Schmitz, D., R.M. Empson, and U. Heinemann. 1995. Serotonin reduces inhibition via 5-HT1a receptors in area CA1 of the rat hippocampal slices in vitro. J. Neurosci. 15: $7217-7225$.

Segal, M. 1990. Serotonin attenuates a slow inhibitory postsynaptic potential in rat hippocampal neurons. Neuroscience 36: 631-641.

Sinton, C.M. and S.L. Fallon. 1988. Electrophysiological evidence for a functional differentiation between subtypes of the 5-HT1 receptor. Eur. J. Pharmacol. 157: 173-181.

Skaggs, W.E., B.L. McNaughton, M.A. Wilson, and C.A. Barnes. 1996. Theta phase precession in neuronal populations and the compression of temporal sequences. Hippocampus 6: 149-172.
Sprouse, J.S. and G.K. Aghajanian. 1987. Electrophysiological responses of serotonergic dorsal raphe neurons to 5-HT1a and 5-HT1b receptors agonists. Synapse 1: 3-9.

1988. Responses of hippocampal pyramidal cells to putative serotonin 5-HT1 a and 5-HT1b agonists: A comparative study with dorsal raphe neurons. Neuropharmacology 27: 707-715.

Toth, K. and T.F. Freund. 1992. Calbindin D28k-containing non-pyramidal cells in the rat hippocampus: Their immunoreactivity for GABA and projection to the medial septum. Neuroscience 49: 793-805.

Vanderwolf, C.H. 1969. Hippocampal electrical activity and voluntary movement in the rat. Electroencephalogr. \& Clin. Neurophysiol. 26: 407-418.

Vertes, R.P. and B. Kocsis. 1997.

Brainstem-diencephalo-septohippocampal systems controlling the theta rhythm of the hippocampus. Neuroscience 81: 893-926.

Vertes, R.P. and G.F. Martin. 1988. An autoradiographic analysis of ascending projections from the pontine and mesencephalic reticular formation and the median raphe nucleus in the rat. J. Comp. Neurol. 275: 511-541.

Vertes, R.P., G.G. Kinney, B. Kocsis, and W.J. Fortin. 1994. Pharmacological suppression of the median raphe nucleus with serotonin 1 a agonists, 8-OH-DPAT and buspirone, produces hippocampal theta rhythm in the rat. Neuroscience 60: $441-451$.

Wilson, M.A. and B.L. McNaughton. 1993. Dynamics of the hippocampal ensemble code for space. Science 261: 993-994.

Ylinen, A., I. Soltesz, A. Bragin, M. Penttonen, A. Sik, and G. Buzsaki. 1995. Sharp-wave associated high-frequency oscillation $(200 \mathrm{~Hz})$ in the intact hippocampus: Network and intracellular mechanisms. J. Neurosci. 14: 30-46.

Received January 26.1999; accepted in revised form March 4, 1999.

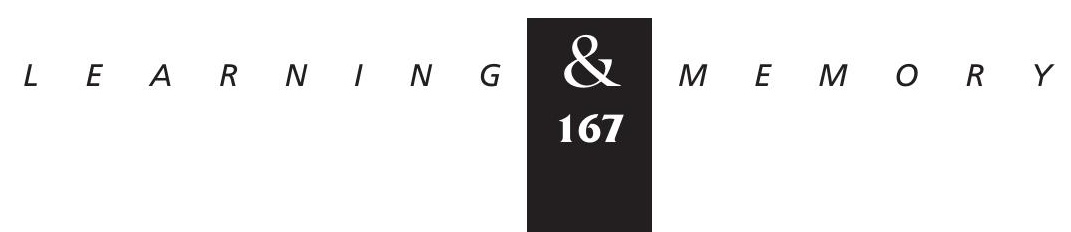




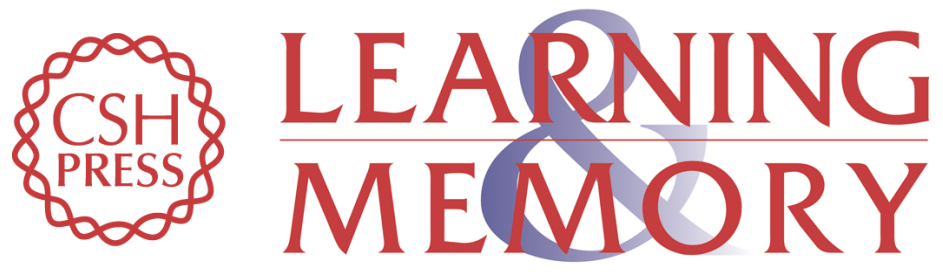

\section{Hippocampal EEG and Unit Activity Responses to Modulation of Serotonergic Median Raphe Neurons in the Freely Behaving Rat}

Douglas A. Nitz and Bruce L. McNaughton

Learn. Mem. 1999, 6:

Access the most recent version at doi:10.1101//m.6.2.153

References This article cites 50 articles, 10 of which can be accessed free at:

http://learnmem.cshlp.org/content/6/2/153.full.html\#ref-list-1

License

Email Alerting Receive free email alerts when new articles cite this article - sign up in the box at the Service top right corner of the article or click here. 